\title{
A simple two-step protocol for the purification of human pancreatic beta cells
}

\author{
M. Banerjee • T. Otonkoski
}

Received: 5 November 2008 / Accepted: 19 November 2008 / Published online: 24 January 2009

(C) Springer-Verlag 2009

\begin{abstract}
Aims/hypothesis Isolated pure human beta cells would be helpful for a number of research purposes. However, lack of beta cell-specific surface antigens has been a major problem. We aimed to develop a simple method for human beta cell isolation based on the initial elimination of ductal cells by their expression of carbohydrate antigen 19-9 (CA19-9), followed by positive selection of beta cells by their expression of polysialic acid-neural cell adhesion molecule (PSA-NCAM).

Methods Cell type-specific expression of CA19-9, NCAM and PSA-NCAM was studied in sections of adult human pancreas and in cultured primary endocrine and exocrine cells. Dispersed human islet cells were purified in two steps, after 4 days of suspension culture, by binding to magnetic microbeads coupled to antibodies against CA19-9 and PSA-NCAM.

Results NCAM expression was detected in ducts and islets in the human pancreas. In contrast, PSA-NCAM immunoreactivity was detected only in islets. PSA-NCAM staining in dispersed cells revealed that the marker is expressed in all endocrine cell types, but not in duct cells. Purification of dispersed islet cells using PSA-NCAM microbeads alone did not completely eliminate contaminating duct cells. However, elimination of the duct cells by CA19-9 microbeads followed by positive sorting of the PSA-NCAM-positive cells in five
\end{abstract}

\footnotetext{
M. Banerjee $(\square) \cdot$ T. Otonkoski $(\square)$

Biomedicum Stem Cell Center, University of Helsinki,

Room C507b, PO Box 63, 00014 Helsinki, Finland

e-mail: meenal2005@gmail.com

e-mail: timo.otonkoski@helsinki.fi

T. Otonkoski

Hospital for Children and Adolescents, University of Helsinki,

Helsinki, Finland
}

consecutive islet preparations resulted in 90 to $98 \%$ pure endocrine cells, of which 89 to $97 \%$ were beta cells.

Conclusions/interpretation We describe a simple and reproducible method for purification of viable human pancreatic beta cells devoid of exocrine acini and ducts.

Keywords Beta cell purification · Human beta cells . Magnetic microbead $\cdot$ Neural cell adhesion molecule

$\begin{array}{ll}\text { Abbreviations } \\ \text { CA19-9 } & \begin{array}{l}\text { carbohydrate antigen 19-9 } \\ \text { neural cell adhesion molecule } \\ \text { NCAM }\end{array} \\ \text { PSA-NCAMsialic acid-neural cell adhesion } \\ & \begin{array}{l}\text { molecule } \\ \end{array}\end{array}$

\section{Introduction}

Unlike isolated rodent pancreatic islets, highly pure human islets are practically impossible to isolate using traditional methods alone. Duct epithelial cells closely associated with islets create a particular problem. Isolated islet preparations used for clinical transplantation contain approximately equal numbers of beta cells and duct cells [1]. Human beta cells can be purified by FACS by virtue of their high zinc content using Newport Green $[2,3]$. The method yields more than $90 \%$ pure beta cells, but depends on expensive instrumentation and considerable flow cytometry expertise. Moreover, an ideal purification method should not involve incorporation of potentially toxic substances into beta cells. A method for rat beta cell purification that has been used successfully for many years is based on flavin adenine dinucleotide and $\mathrm{NAD}(\mathrm{P}) \mathrm{H}$ autofluorescence [4]. However, 
although this method is efficient in rodent beta cells, it has been difficult to apply for human beta cell purification.

Within the pancreas, neural cell adhesion molecule (NCAM) is known to play an important role in cell-cell adhesion between the endocrine cells [5]. Attachment of sialic acid polymers through $\alpha-2,8$ binding to NCAM leads to formation of a polysialylated form of the molecule, namely polysialic acid-NCAM (PSA-NCAM) with modified adhesive properties [6]. PSA-NCAM is expressed in pancreatic beta cells of adult rats and has been used to sort rat beta cells [7].

Here we report on a two-step method for purification of human beta cells using magnetic microbeads. The method is based on the elimination of ductal cells using an antibody against carbohydrate antigen 19-9 (CA19-9), an antigen expressed on the luminal surface of pancreatic ducts $[8,9]$, followed by positive selection of cells expressing PSA-NCAM.

\section{Methods}

Human islets Islets were isolated as described earlier [10]. Informed consent was obtained from tissue donors and institutional Ethics Committees in Uppsala and Helsinki approved all procedures. Upon arrival, islets were cultured for 4 days in non-adherent culture dishes (Barloword Scientific, Stone, UK) in serum-free Ham's F10 (10 mmol/l glucose; Sigma, St Louis, MO, USA) containing $0.5 \%$ bovine serum albumin (Sigma).

Islet dispersion Initial cell clusters were dissociated with $0.16 \mathrm{mg} / \mathrm{ml}$ trypsin and $0.1 \mathrm{mmol} / 1$ EDTA and filtered through a $70 \mu \mathrm{m}$ nylon mesh (Becton Dickinson Biosciences, Bedford, MA, USA).

Cell sorting and culture For direct cell sorting, dispersed islets were incubated for 15 min with microbeads conjugated to PSA-NCAM (Miltenyi Biotech, Auburn, CA, USA). After a brief centrifugation at $300 \mathrm{~g}$, cells were incubated for another 15 min with goat anti-mouse IgG microbeads (1:5; Miltenyi Biotech) in $250 \mu \mathrm{l}$ of phosphate buffered saline. Cell separation was carried out on a MiniMACS magnetic cell separation system according to manufacturer's instructions (Miltenyi Biotech).

For two-step cell sorting, dispersed islet cells were first incubated for $5 \mathrm{~min}$ at $4^{\circ} \mathrm{C}$ with mouse anti-human CA19-9 antibody and cell separation carried out as above. Negative cell fraction was immediately used for PSA-NCAM sorting as described above. All procedures of cell sorting except incubations were done at room temperature to minimise cell death [9].
Prior to immunostaining, purified cells were cultured for 4 days in 48-well dishes (Nunc, Roskilde, Denmark) in RPMI 1640 medium (Invitrogen, Carlsbad, CA, USA) containing 10\% FCS (vol./vol.; PromoCell, Heidelberg, Germany).

Immunostaining Cells were fixed in 4\% paraformaldehyde (wt/wt) and permeabilised with $0.1 \%$ Triton X-100 (vol./vol.). Pancreatic sections were deparaffinised and rehydrated before staining. Microwave treatment in citrate buffer was necessary to retrieve antigenicity of NCAM, CA19-9 and PSA-NCAM.

Non-specific binding was blocked by preincubation in 5\% normal serum (vol./vol.; Zymed, San Francisco, CA, USA) of the species in which secondary antibody was raised. Specimens were incubated for $1 \mathrm{~h}$ with primary antibody followed by secondary antibody incubation for $45 \mathrm{~min}$. The following primary antibodies were used: guinea pig antiporcine insulin 1:200, rabbit anti-human glucagon 1:300, rabbit anti-human somatostatin 1:300, rabbit anti-human pancreatic polypeptide 1:300 (a mixture of glucagon, somatostatin and pancreatic polypeptide antibodies was used to simultaneously label alpha, delta and pancreatic polypeptide cells), rabbit anti-human chromogranin-A 1:500, mouse antihuman CK19 1:200 (all from Dako, Glostrup, Denmark); rabbit anti- $\alpha$-amylase (1:300; Sigma); anti-PSA-NCAM (1:100; mouse monoclonal IgM; Chemicon, Billerica, MA, USA); anti-CA19-9 (1:100; mouse monoclonal; Novocastra, Newcastle upon Tyne, UK); and rabbit anti-NCAM 1:50 (kindly provided by V. Cirulli, The Whittier Institute for Diabetes, San Diego, CA, USA). Alexa fluor-conjugated goat anti-guinea pig, donkey anti-rabbit and donkey anti-mouse (1:300; all from Invitrogen), and FITC-conjugated rabbit antimouse IgM (Jackson Immuno Research Laboratories, West Grove, PA, USA) were used as secondary antibodies.

\section{Results and discussion}

Expression of CA19-9, NCAM and PSA-NCAM in adult human pancreas As expected, CA19-9 immunoreactivity was found in all duct cells but not in islets (Fig 1b). In contrast, PSA-NCAM immunoreactivity was found to be restricted to islets (Fig 1c). NCAM showed a wide expression pattern, both on ducts and islets (Fig 1a). To further examine the expression of PSA-NCAM in freshly isolated cells, islets were dispersed into single cells and double-stained for PSA-NCAM and islet hormones. Colocalisation of PSA-NCAM immunoreactivity with insulin and other islet hormones showed that it is expressed not only in beta cells, but also in other endocrine cells. No PSA-NCAM signal was detected in CK19-positive duct cells (Fig 1d). These results are different from a previous report [7], where PSA-NCAM expression was restricted to 
a

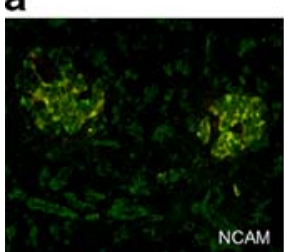

NCAM

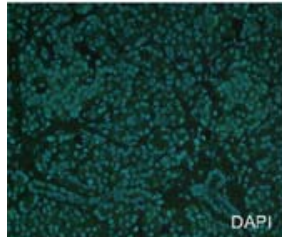

DAPJ
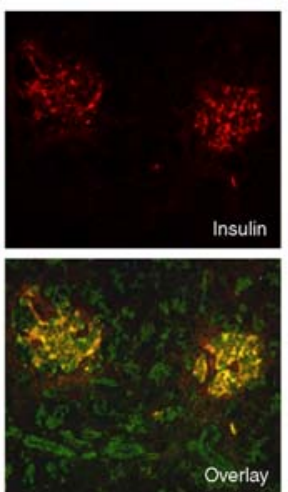

d
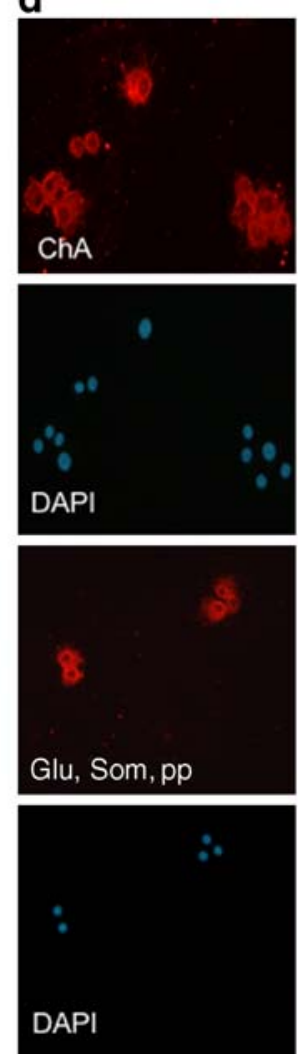

b
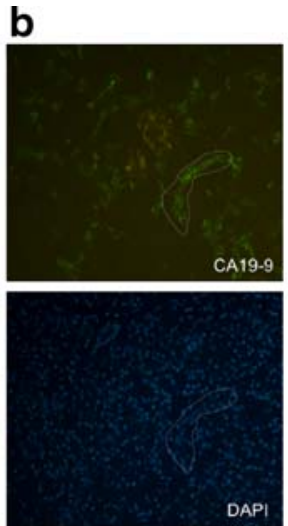

DAPI
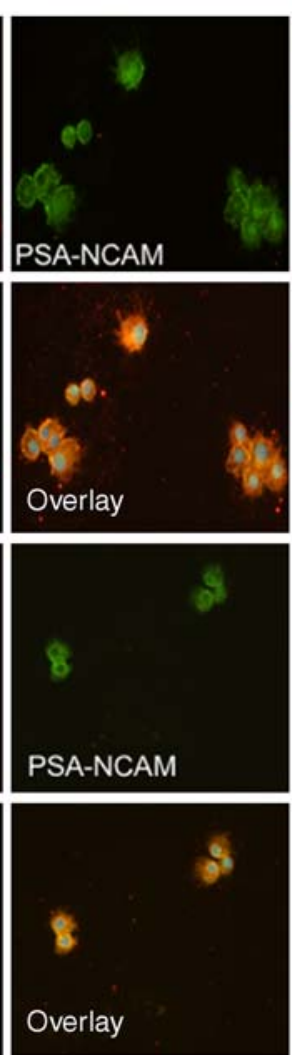

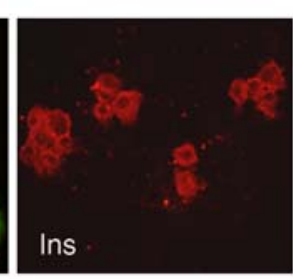

C
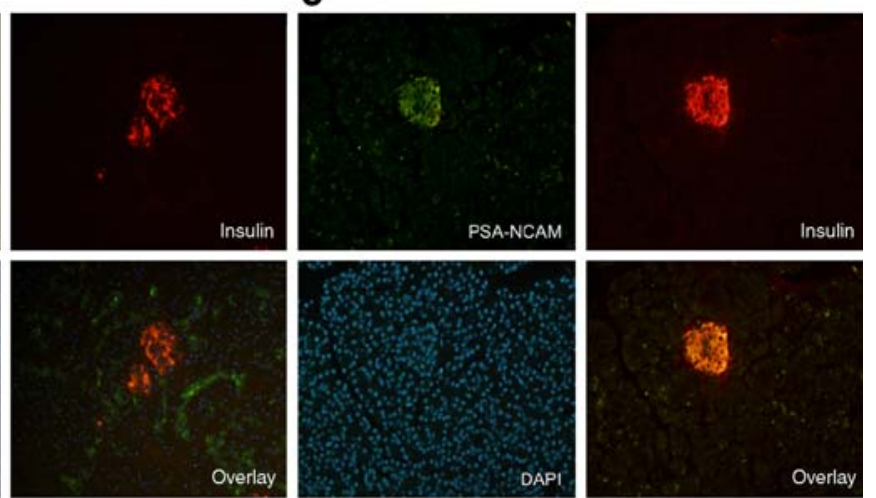
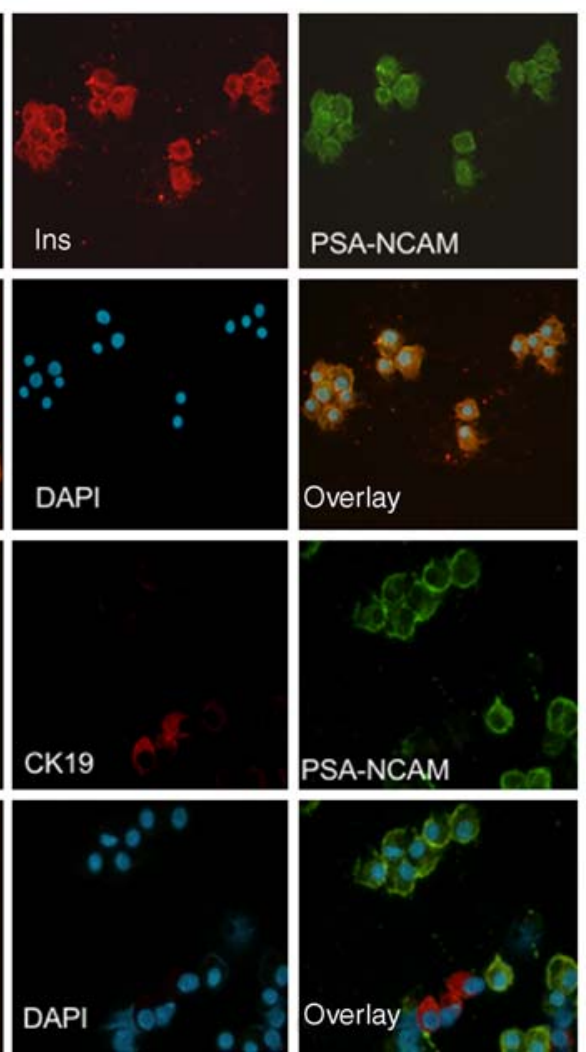

Fig. 1 Human pancreatic sections were immunostained for NCAM (a), CA19-9 (b) and PSA-NCAM (c) (all green), together with insulin (red). Nuclei were labelled with DAPI. Pancreatic duct next to an islet is defined by a dotted line (b). d PSA-NCAM immunostaining in freshly isolated islet cells together with endocrine and duct cell

markers. Duct cells were labelled with cytokeratin-19, whereas endocrine cells were labelled with chromogranin-A, insulin and a combination of antibodies against glucagon (glu), somatostatin (som) and pancreatic polypeptide (pp) to mark alpha, delta and pancreatic polypeptide cells

beta cells only in rats. This is another example of interspecies variation between rodent and human islets.

Two-step purification of human endocrine cells Human islets of various degrees of purity (35 to $90 \%$ dithizonepositive) were cultured in non-adherent Petri dishes prior to purification. The islets were dispersed into single cells prior to purification. In initial trials, we applied PSA-NCAM microbeads directly. However, many contaminating duct cells were found in the positive fraction obtained by PSA-NCAM sorting alone. A possible reason is that the dissociation did not always result in single cells, but contained many cell doublets and triplets. Therefore it is likely that endocrine cells attached to duct cells led to significant duct cell contamination in the positive fraction. Attempts to enhance islet dissociation led to unacceptable cell loss, which led us to test the alternative approach of depleting the number of ductal cells. 
Table 1 Immunocytochemical analysis of cell fractions after the two-step affinity purification in five consecutive human islet preparations

\begin{tabular}{cccccc}
\hline Pancreas number & Initial islet purity (\%) & Insulin-positive cell count (\%) & & \multicolumn{2}{l}{ Sorted cells (\%) } \\
\cline { 4 - 6 } & & & Ch A & Insulin & Other endocrine $^{\mathrm{c}}$ Other cells $^{\mathrm{d}}$ \\
\hline 1 & 35 & 7.5 & 90 & 80 & 10 \\
2 & 60 & 22 & 94 & 85 & 9 \\
3 & 70 & ND & 96 & 90 & 6 \\
4 & 90 & ND & 98 & 94 & 4 \\
5 & 90 & 98 & 95 & 4 \\
\hline
\end{tabular}

${ }^{\mathrm{a}}$ Dithizone staining

${ }^{\mathrm{b}}$ In initial islet preparation by immunostaining

${ }^{\mathrm{c}}$ Based on positivity using a cocktail of antibodies against glucagon, somatostatin and pancreatic polypeptide

${ }^{\mathrm{d}}$ Other cells included: amylase or CK19-positive cells plus the cells that do not express any of the antigens studied

Ch A, chromogranin-A; ND, not done

Based on immunocytochemical analysis, crude islet preparations (purity ranging from 35 to $90 \%$ by dithizone staining) prior to purification contained $40 \pm 28.2 \%$ chromogranin- A-positive cells, $27.5 \pm 23.2 \%$ beta cells, $21 \pm 19.7 \%$ other endocrine cells and $27.5 \pm 17.6 \%$ other cell types (amylase-positive exocrine cells or CK19-positive ductal cells). Depletion of CA19-9 cells prior to PSA-NCAM purification led to the loss of only $2 \pm 0.5 \%$ endocrine cells. However, the purity of beta cells in the final fraction was greatly enhanced.

An immunocytochemical analysis of the cell populations derived from the two-step purification in five consecutive human islet preparations is presented in Table 1. Nearly all purified cells expressed PSA-NCAM, the antigen used for
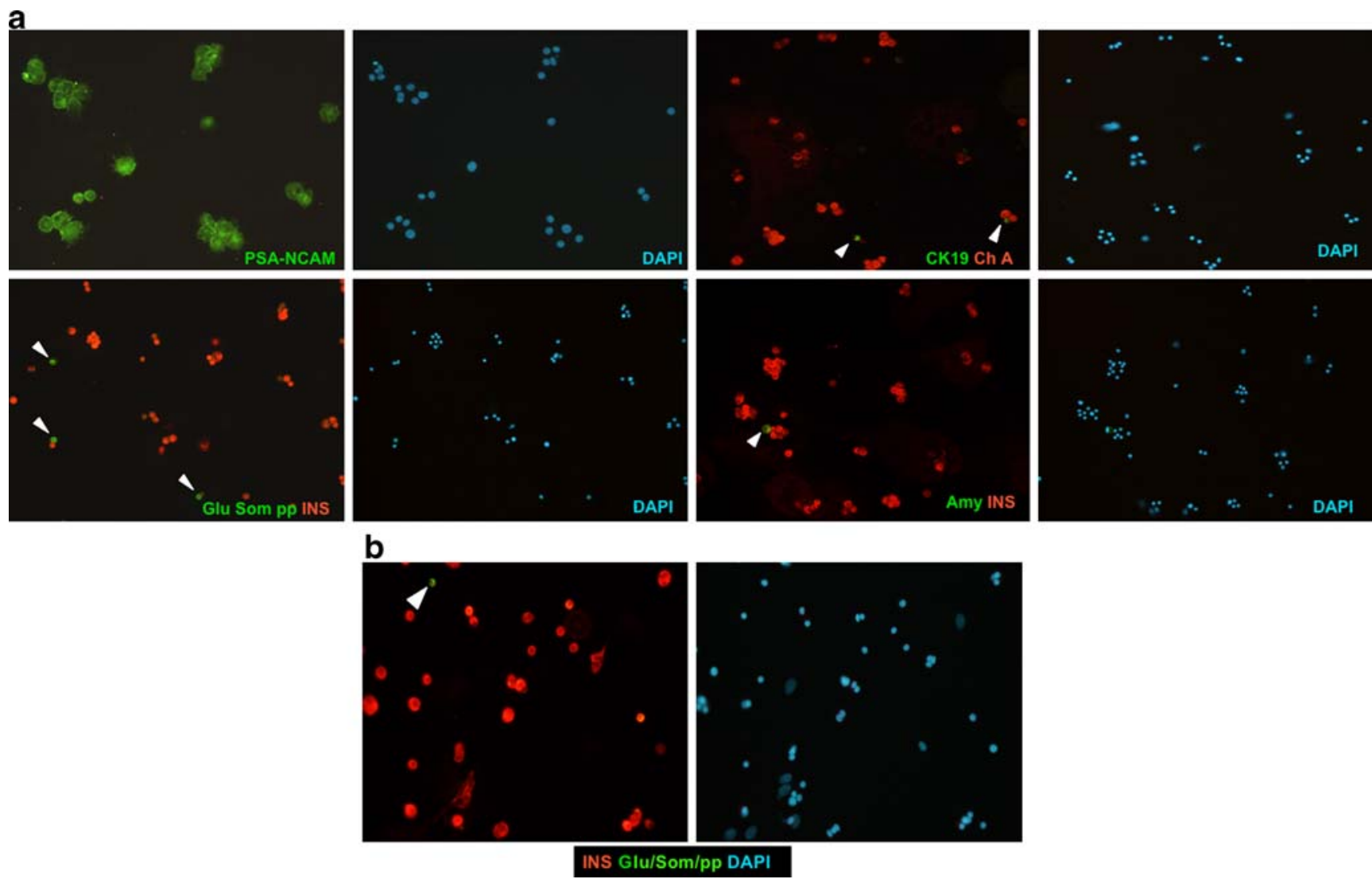

Fig. 2 Immunocytochemical analysis of the affinity-purified cell fraction obtained from the two-step protocol. The cells were stained for PSA-NCAM (green), the label used for purification (a). Double immunolabelling of insulin (INS) or chromogranin-A (Ch A) in red, together with CK19, amylase (Amy) and non-beta islet cells (a cocktail of antibodies against glucagon [Glu], somatostatin [Som] and pancreatic polypeptide [pp]) in green shows the high purity of beta cells. b The purified cells were cultured for 4 days and then doublestained for insulin (red) and non-beta islet cells (green, arrowhead) 
sorting. The great majority of cells were found to be beta cells as shown by chromogranin-A and insulin immunoreactivity. Only few cells were positive for other islet hormones (labelled by a mixture of antibodies against glucagon, somatostatin and pancreatic polypeptide) and only single cells expressed amylase or CK19 (Fig 2a). The higher the endocrine cell content was initially, the higher the purity of beta cells in the sorted cell fraction (Table 1). Based on absolute counts of insulin-positive cells in the pre-purification vs purified fraction, the recovery of beta cells was $\geq 45 \%$ using this method. The protocol is highly selective for beta cells, as it allows enrichment of the insulin-positive cell population from $27.5 \pm 23.2$ to $88.8 \pm$ $6.3 \%$, with significant depletion of other endocrine cells from $21 \pm 19.7$ to $6.6 \pm 2.7 \%$. It thus appears that although PSA-NCAM immunoreactivity is detected in all islet cell types, the affinity of the antibody is much higher against beta cells. In the rat, high PSA-NCAM specifically characterises a subpopulation of functionally active beta cells [7]. It is thus possible that the observed enrichment is due to the selection of a population of beta cells with high PSANCAM expression.

More than $95 \%$ of the sorted cells excluded trypan blue and adhered to tissue culture plates within $24 \mathrm{~h}$. Cells were maintained for 4 days in culture prior to immunostaining. The majority of cultured cells expressed insulin with little contamination by other endocrine cells (Fig 2b), further demonstrating selective enrichment of viable beta cells using this purification method.

Conclusions We have previously used magnetic cell sorting to obtain non-endocrine CK19-enriched cell populations by eliminating NCAM-positive cells [10]. However, the use of CA19-9 microbeads allows much more efficient purification of ductal cells, as described previously [9, 11]. Using the combination of microbeads targeted against CA19-9 and PSA-NCAM, it is now possible to isolate highly purified populations of ductal and beta cells in a simple two-step procedure. Cell purification based on magnetic sorting is easy to adopt and does not involve expensive instruments. Although PSA-NCAM is expressed by all endocrine cells in the human islet, the microbeads coupled to anti-PSA-NCAM antibody bind preferentially to beta cells, thus allowing selective enrichment of beta cells. Our results suggest that the two-step purifi- cation described here is a highly reproducible and easy method for the effective purification of isolated human beta cells.

Acknowledgements The authors are grateful to the personnel of the central laboratory of the Nordic Network for Clinical Islet Transplantation for providing human islets. These studies were funded by the European Union (STREP SAVEBETA, contract no. 036903) in the 6th Framework Program of the European Community, the Academy of Finland, Juvenile Diabetes Research Foundation, the Sigrid Jusélius Foundation and the Research Funds of Helsinki University Central Hospital (TYH7216).

Duality of interest The authors declare that there is no duality of interest associated with this manuscript.

\section{References}

1. Street CN, Lakey JR, Shapiro AM et al (2004) Islet graft assessment in the Edmonton Protocol: implications for predicting long-term clinical outcome. Diabetes 53:3107-3114

2. Lukowiak B, Vandewalle B, Riachy R et al (2001) Identification and purification of functional human beta-cells by a new specific zinc-fluorescent probe. J Histochem Cytochem 49:519-528

3. Parnaud G, Bosco D, Berney T et al (2008) Proliferation of sorted human and rat beta cells. Diabetologia 51:91-100

4. Pipeleers DG, in't Veld PA, Van de Winkel M, Maes E, Schuit FC, Gepts W (1985) A new in vitro model for the study of pancreatic A and B cells. Endocrinology 117:806-816

5. Cirulli V, Baetens D, Rutishauser U, Halban PA, Orci L, Rouiller DG (1994) Expression of neural cell adhesion molecule (N-CAM) in rat islets and its role in islet cell type segregation. J Cell Sci 107:1429-1436

6. Rutishauser U, Acheson A, Hall AK, Mann DM, Sunshine J (1988) The neural cell adhesion molecule (NCAM) as a regulator of cell-cell interactions. Science 240:53-57

7. Bernard-Kargar C, Kassis N, Berthault MF, Pralong W, Ktorza A (2001) Sialylated form of the neural cell adhesion molecule (NCAM): a new tool for the identification and sorting of beta-cell subpopulations with different functional activity. Diabetes 50 (Suppl 1):S125-S130

8. Gmyr V, Belaich S, Muharram G et al (2004) Rapid purification of human ductal cells from human pancreatic fractions with surface antibody CA19-9. Biochem Biophys Res Commun 320:27-33

9. Yatoh S, Dodge R, Akashi T et al (2007) Differentiation of affinity-purified human pancreatic duct cells to beta-cells. Diabetes $56: 1802-1809$

10. Gao R, Ustinov J, Korsgren O, Otonkoski T (2005) In vitro neogenesis of human islets reflects the plasticity of differentiated human pancreatic cells. Diabetologia 48:2296-2304

11. Ichii H, Miki A, Yamamoto $\mathrm{T}$ et al (2008) Characterization of pancreatic ductal cells in human islet preparations. Lab Invest 88:1167-1177 